\title{
Optimality Modeling in a Suboptimal World*
}

\author{
Angela Potochnik
}

\begin{abstract}
The fate of optimality modeling is typically linked to that of adaptationism: the two are thought to stand or fall together (Gould and Lewontin, 1979; Orzack and Sober, 1994). I argue here that this is mistaken. The debate over adaptationism has tended to focus on one particular use of optimality models, which I refer to here as their strong use. The strong use of an optimality model involves the claim that selection is the only important influence on the evolutionary outcome in question and is thus linked to adaptationism. However, biologists seldom intend this strong use of optimality models. One common alternative that I term the weak use simply involves the claim that an optimality model accurately represents the role of selection in bringing about the outcome. This and other weaker uses of optimality models insulate the optimality approach from criticisms of adaptationism, and they account for the prominence of optimality modeling (broadly construed) in population biology. The centrality of these uses of optimality models ensures a continuing role for the optimality approach, regardless of the fate of adaptationism.
\end{abstract}

Keywords: adaptationism, behavioral ecology, evolution, natural selection, optimality modeling, population biology

${ }^{*}$ Forthcoming in Biology and Philosophy, 2008. 
Criticisms of adaptationism have been well-articulated and assimilated into the thinking of most population biologists and philosophers of biology, and optimality modeling is typically regarded to be adaptationism's henchman. Consequently, when one defends the value of optimality models to evolutionary biology, the first question posed is often, "but what about all of the problems with adaptationism?" My aim here is to decouple optimality modeling from adaptationism.

Distinct theses are lumped under the term "adaptationism" (Orzack and Sober, 1994; Godfrey-Smith, 2001), but roughly, the idea is that selection is the only important influence on evolutionary change (Orzack and Sober, 1994) and that, for this reason, phenotypic traits typically attain optimal values (Gould and Lewontin, 1979). Gould and Lewontin's seminal paper (1979) formulated these criticisms in terms of optimality modeling, and others have followed suit. Optimality models - equilibrium models that represent the evolution of a phenotypic trait as a function of the relative fitnesses of the trait values - are thought to presuppose the ubiquity and omnipotence of selection and to ignore the possibility of constraints and other non-selective evolutionary factors. These models are consequently held to be suspect.

In contrast, I argue in this paper that the legitimate and fruitful use of optimality models does not require the vindication of adaptationism. The situation is that of the baby and the bathwater. Criticisms of adaptationism should be taken seriously (as they in fact are), and these criticisms do have implications for optimality modeling. Yet optimality modeling does not require the truth of adaptationism in order to be a valuable tool for population biology. Only one particular use of optimality models - what I call their strong use - is directly linked to adaptationism. The strong use of an optimality model involves the claim that selection is the only important factor influencing the evolutionary outcome in question.

Interestingly, whereas most philosophical literature focuses on this strong use, population biologists oftentimes employ the optimality approach for other purposes. Optimality models 
are frequently used simply to represent the role of selection in bringing about an evolutionary outcome, and even just to preliminarily investigate the mere possibility of selective influence on a trait. Neither of these weaker uses of optimality models involves the claim that selection is the only important influence on the evolutionary outcome that is modeled. Thus, although the fates of the strong use of optimality models and adaptationism are tightly linked, the same is not true for other uses of optimality models. Weaker uses of optimality models do not require the vindication of adaptationism to establish their legitimacy. Because these weaker uses are quite common in - and quite valuable to-population biology, critiques of adaptationism have a limited impact on the well-foundedness of the optimality approach.

\section{Different Uses of Optimality Models}

The optimality approach provides a way to model evolution by natural selection purely phenotypically, without directly representing the system of genetic transmission. Optimality models proceed according to the following schema. One determines the range of possible trait values for the phenotype of interest and the fitness function that relates these phenotypic trait values to their success in the present environment. Based on this information, an optimality model predicts which trait value(s) would predominate in the population as a result of selection, given enough time in the current environment for an equilibrium to be reached. ${ }^{1}$

Different claims might be made about the relationship between an optimality model and the actual evolutionary outcome. For instance, the assumption that selection is the sole

\footnotetext{
${ }^{1}$ Understood in this way, the optimality approach also includes game-theoretic models, which are used when trait fitnesses are frequency-dependent. The fitness functions used in game-theoretic models take into account not only the environment, but also the trait values (strategies) of the other members of the population. For this reason, game-theoretic models often do not predict a single optimal trait value, but an optimal distribution of trait values in the population. Some game-theoretic models even predict that there will not be a stable distribution of trait values, but a continual cycle of change (Hofbauer and Sigmund, 1998). My points in this paper apply to this broad class of models, for they pertain to all equilibrium models that do not represent genetic transmission, regardless of whether a single optimal trait value is predicted.
} 
evolutionary factor at work and that sufficient time has passed in the current environment grounds the claim that a successful optimality model's prediction will perfectly match the observed trait value(s). Relaxing one or more of these assumptions results in various weaker claims, e.g., that the model's prediction would be fully accurate if enough time had passed. Different uses of optimality models are distinguished by these different types of claims made about the relationship between an optimality model and a target evolutionary outcome.

What I have termed the strong use of an optimality model occurs when a model is taken to represent all important influences on the evolution of the trait modeled. The claim that an optimality model represents all important causes of the evolutionary outcome in question posits the tightest possible connection between the optimality model and the evolutionary outcome. In order for this claim to be true - that is, in order for an optimality model to represent all important causes of the evolutionary outcome - several conditions must be met. The model's fitness function and the range of possible trait values must be fully accurate in order for the dynamics of selection to be accurately represented. Sufficient time must have passed for the trait to reach equilibrium in order for these selection dynamics to have effected the expected trait value(s). Finally, natural selection must be the only important influence on the evolutionary outcome in question. This last condition ensures that the optimality model's representation of the selection dynamics is exhaustive of the evolutionary influences on the outcome. Put more simply, this ensures that the optimality model tells the whole story.

Implicit in this conception of the requirements for the strong use of optimality models is the idea that selection is the only evolutionary factor that optimality models represent. One might object that optimality models actually do represent other evolutionary factors via incorporating constraints. I will not provide a decisive argument against such a view here; what is required for a model to represent an influence-as opposed to neglecting or black-boxing it - is a complicated question. Nonetheless, at least two considerations suggest 
that selection is the only evolutionary factor represented by optimality models. First, broad physical and biological constraints always limit the range of evolutionarily possible trait values, so every optimality model must incorporate these types of broad constraints. Yet selection is sometimes considered to be the only evolutionary factor at work, despite the ubiquity of background constraints. This is possible because these types of broad, background constraints are not counted as distinct evolutionary factors, but simply are taken to reflect the realm of physical and biological possibility. So at least for these types of constraints, their incorporation into optimality models is not taken to constitute the representation of additional evolutionary factors. Second, if optimality models only represent selection, then this maintains a tight connection between the strong use of optimality models and adaptationism. The tradition of linking optimality modeling to adaptationism suggests that some use of optimality models is valuable to determining the truth of the various adaptationist claims, and it seems that the strong use of optimality models is best positioned to play this role. I thus proceed on the assumption that optimality models do not actually represent non-selective evolutionary factors, even when they take these factors into account by incorporating constraints.

The strong use of an optimality model thus amounts to a claim about representation: that the model accurately represents the selection dynamics, and that there are no other important evolutionary factors to represent (for the target evolutionary outcome in question). Unsurprisingly, the strong representational role intended with this use of optimality models results in stringent standards for model testing. If the model's predictions are inaccurate, even to a small degree, then this is evidence that selection is misrepresented or that there is another evolutionary influence on the outcome. From this it would follow that the strong use of the optimality model in question is not successful - the model does not represent all important evolutionary influences. Additional evidence regarding the success of the strong use of an optimality model comes from comparing the optimality model's pre- 
dictions with the predictions of models that represent additional evolutionary factors, such as genetic transmission and drift. If selection is the only important evolutionary influence on an outcome, then the optimality model's predictions should be at least as accurate as those of these alternate types of models. Even observed variation around the predicted trait value(s) may indicate a non-selective evolutionary influence and, consequently, the failure of the optimality model in its strong use. The stringent evidential requirements that accompany the strong use of optimality models are precisely those that have been identified as important in generating support for adaptationism (Orzack and Sober, 1994; Brandon and Rausher, 1996; Orzack and Sober, 1996).

Most of the philosophical discussion of optimality modeling regards the nature of the connection between the optimality approach and adaptationism. Throughout this discussion, the strong use of optimality models is implicitly assumed. For instance, Orzack and Sober state that

The rationale for the focus on phenotypes is the notion that natural selection will overcome any genetic or developmental constraints on an evolutionarily important trait $(1994,362)$.

That is, the justification given for optimality modeling-i.e., for focusing on phenotypic change directly and ignoring the details of genetic transmission and development - is that natural selection is the only important influence on the evolution of many traits (the "evolutionarily important" ones). If selection is the only important evolutionary influence, then genetic and developmental details can be safely ignored. This rationale is linked to the strong use of optimality models, in which an optimality model's representation of selection is taken to give a complete picture of the evolutionary influences at work.

Yet the strong use of optimality models is not the only way, nor even the standard way, that the optimality approach is employed in population biology. Optimality models are routinely constructed for other purposes, purposes that involve weaker claims about their 
representational capacities. As outlined earlier in this section, different claims can be made about the relationship between an optimality model and a target evolutionary outcome, and these different claims distinguish different uses of optimality models. The strong use of an optimality model involves the strongest representational claim: that the model accurately represents all evolutionary dynamics. A distinct use of an optimality model arises with the weaker claim that the model represents the role of natural selection in bringing about the evolutionary outcome. Absent is the claim that the model represents all evolutionary influences. I call this the weak use of optimality models.

This weaker representational role of optimality models results in less stringent criteria for a model's success. In order to succeed in its weak use, an optimality model must accurately represent the selection dynamics involved in producing the target evolutionary outcome, but a model may still be successful even if factors besides selection influence the outcome. Optimality models can reflect the effects of some non-selective influences by altering the fitness function and/or range of possible trait values in response to the existence of genetic, phylogenetic or developmental constraints. Other constraints, genetic drift, and insufficient time for the trait to reach equilibrium may be unaccounted for, which can lead to some deviation between the model's prediction and the observed trait value(s). Within limits, such deviation does not impede the success of an optimality model. In its weak use, an optimality model must accurately represent selection, but if selection is only part of the evolutionary story, then observed trait values may vary from the values the optimality model predicts. ${ }^{2}$

Of course, the dynamics of the optimality model must correspond to the actual evolutionary dynamics in some respects, viz., the model must accurately represent the dynamics of selection. Most basically, in order for this correspondence to occur, it must be the case that selection influences the evolutionary outcome in question. Additionally, the optimality

\footnotetext{
${ }^{2}$ If there is a large amount of deviation between predicted and observed trait values, then an optimality model may be taken to fail, even in the weak use outlined here. This point is discussed in Section 2.
} 
model's fitness function must accurately represent the nature of this selective influence; the range of possible trait values used in the model must be accurate; and enough time must have passed for selection to have had a chance to influence the trait in question. If the optimality model is successful in these regards, then there will be at least some qualitative agreement between the model's predictions and the observed trait values. When the influence of non-selective factors is small, or when constraints are used to account for the effects of non-selective influences, an optimality model's predictions likely will be quite accurate.

The successful weak use of an optimality model does not ensure that the evolutionary outcome occupies a local optimum (or an evolutionary stable state; see footnote 1). As I pointed out above, the strong use requires perfect correspondence between predicted and observed trait values. In other words, for the strong use of an optimality model to be successful, the optimizing assumption must be true: the trait must occupy the predicted local optimum. In contrast to this, the weak use simply requires establishing that selection influences the trait in question, and that the optimality model accurately represents this influence. The trait may very well not occupy a local optimum.

One way to establish the intended use of an optimality model is thus to consider the ways in which the model is tested. It is telling that the optimizing assumption is not often subject to test. Population biologists typically do not devote much effort to establishing whether the observed outcome represents a local optimum - that is, whether selection has driven the population to an optimum, unimpeded by other evolutionary factors. Instead, the emphasis is placed on testing the particulars of the fitness function and on confirming assumptions regarding the heritability of the trait, the range of possible trait values, the existence of selection, and the constancy of the environment. The success of these model components is necessary for an optimality model to represent the role of selection, but this alone does not confirm the optimizing assumption. More is required to generate support for the strong use of an optimality model: the perfect correspondence of predicted optimum and observed 
trait value(s) is needed demonstrate that selection is the only important evolutionary factor. The common tests of optimality models thus only underwrite what I have termed optimality models' weak use. Consequently, it appears that biologists who employ optimality models seldom intend the strong use of these models.

Seger and Stubblefield point out this connection between testing and the uses to which optimality models are put. They say,

In fact, the aims of real optimization studies are rarely to see how close a phenotype is to its optimal state... The hypothesis under test is usually not that a phenotype is optimal, but instead that the specific assumptions embodied in the model (e.g., the sources of selection, and the constraints) could account at least in principle for the evolution of the phenotypes under study $(1996,109,110)$.

This observation highlights that the strong use of optimality models - establishing whether and the degree to which a phenotypic trait is optimal - is seldom the aim of the optimality approach. It also raises a distinct point which deserves mention. The weak use of optimality models upon which I have focused involves the claim that an optimality model accurately represents the actual selection dynamics. There is another common use of optimality models that is still weaker: optimality models are also used to develop and explore adaptive hypotheses at preliminary stages of investigation, when little is known about the evolutionary factors at work. Used in this capacity, the aim of optimality modeling is merely to represent possible selection dynamics, and the standards for success are correspondingly lower. ${ }^{3}$ This may be what Seger and Stubblefield have in mind when they say that optimality models are used to evaluate whether specific assumptions about selection and constraints, etc., could account at least in principle for the target evolutionary outcome.

\footnotetext{
${ }^{3}$ The members of Joan Roughgarden's lab helped me grasp this point. Also, note that the exploration of possible evolutionary dynamics is consistent with the eventual aim of understanding the actual evolutionary dynamics.
} 
Thus, despite the fact that most philosophical discussion of optimality models presumes the strong use of these models, population biologists often employ optimality models in weaker capacities. Because the strong use of an optimality model involves the claim that the model represents all important evolutionary influences, the frequency of success for the strong use is directly related to the fate of adaptationism. This is what Orzack and Sober (1994), e.g., have in mind. In contrast, weaker uses of optimality models are not threatened by critiques of adaptationism. One prominent example is the weak use upon which I have focused here. In this weak use, an optimality model is used simply to represent the dynamics of selection, which may comprise only one part of the evolutionary dynamics.

\section{Lessons from the Adaptationist Critique}

Although weaker uses of optimality models can be decoupled from adaptationism, critiques of adaptationism certainly do have implications for the optimality approach. These critiques have forcefully made the point that the ubiquity and omnipotence of selection cannot be presumed. This and related insights have several implications for optimality models in the various representational capacities in which they are employed.

First, it is a familiar point that optimality models should be carefully developed to incorporate any existing constraints (Maynard Smith, 1982). I said above that in their weak use, optimality models may not incorporate all constraints that influence the evolutionary outcome. The aim of the weak use is to accurately represent the dynamics of natural selection, and since constraints are used to stand in for other parts of the evolutionary story, optimality models can succeed at this aim even if they fail to reflect all extant constraints. Nonetheless, if an optimality model neglects constraints that result in trait value(s) radically different from the predicted optimum, then this model is in some sense a failure. The model represents the dynamics of selection, but it represents these dynamics as they would work 
in quite different circumstances than in fact obtain-namely, in conjunction with fewer or different non-selective evolutionary factors. Such an optimality model is of limited interest and would be much improved by the incorporation of significant constraints.

As a simple example, consider the evolution of kangaroo locomotion. Because kangaroos travel by a series of leaps, changes that improve their ability to move in this way are selectively advantaged and will likely evolve. Imagine, though, that bipedal running is even more advantageous. In contrast to improved leaping ability, it is nonetheless highly unlikely that kangaroos would evolve the ability to run. This is because initial changes in movement in the transformation to bipedal running would be significantly maladaptive (Maynard Smith et al., 1985). If bipedal running is selectively advantaged in a kangaroo population, then an optimality model that reflects this in some sense accurately represents selection pressures. Yet because initial changes in the direction of bipedal running are disadvantageous, bipedal running is highly unlikely to evolve, so a model that represents the selective advantage of running would be of limited or no interest. This example drives home the point that optimality models are used to locate local optima. In order for the information gained from an optimality model to be valuable, the predicted optimum should be local for the population(s) of interest. This is why an accurate range of possible trait values is important even for the weak use of optimality models. It also justifies the requirement that constraints with a tremendous influence on the outcome be incorporated in optimality models, even in their weak use.

A second consequence of the point that the ubiquity and omnipotence of selection cannot be presumed is that it must be established whether a trait is subject to selection in the first place. Gould and Lewontin (1979) catalog several possible non-selective sources of evolutionary change. These include scenarios in which natural selection is not a factor at all - such as the founder effect, the fixation of a less favorable allele due to drift, and the loss of favorable mutations while still rare in the population. Additionally, selection acting on 
other, correlated traits might also drive the evolution of a trait, as happens with pleiotropy and allometry. Even for the weak use of optimality models that I describe, it must be established that the trait in question is in fact subject to selective pressures.

As one would expect, though, other implications of critiques of adaptationism are particular to the strong use of optimality models. Because the strong use of an optimality model involves the claim that the optimality model represents all important influences on the outcome, selection must be the only evolutionary factor that significantly influences the target evolutionary outcome. This has several implications for the strong use of optimality models. First, because an optimality model must provide an accurate representation of all of the evolutionary dynamics (viz., the selection dynamics) to succeed in its strong use, the model's predicted local optimum must exactly match observed trait value(s). This requirement was discussed above: the model must predict the observed distribution of trait values, and any unpredicted variation must be accounted for (Orzack and Sober, 1994; Brandon and Rausher, 1996; Orzack and Sober, 1996). Establishing this exact correspondence is needed to confirm the model's optimizing assumption, that is, that the trait occupies the local optimum.

Each of the other model components also must be carefully tested for the strong use of an optimality model. As Gould and Lewontin (1979) point out, unmotivated assumptions can lead to inaccuracies in a model, which can in turn conceal a disparity between predicted and observed trait values - that is, the failure of optimality. The only way to ensure that an incorrect representation of the evolutionary dynamics does not conceal the failure of the optimizing assumption is to rigorously test the postulated dynamics to ensure their accuracy. Components of the model that should be tested include, e.g., the details of the fitness function; the assumption that there is phenotypic variability for the trait in question; and the assumption that this variation is heritable. ${ }^{4}$

\footnotetext{
${ }^{4}$ Lloyd (1988) distinguishes between three types of confirmation: fit between predicted and observed
} 
An example of using ecological information to test a postulated fitness function is provided by Parker's (1970) work on the amount of time the male dung fly (Scatophaga stercoraria) spends at a cowpat - the species' mating location-before moving on to a new one (Maynard Smith, 1982). When Parker found that male stay times are exponentially distributed, he tested rates of females' arrival to cowpats of various ages and the average amount of time it takes a male fly to find a new cowpat. These measurements confirmed that the exponential distribution of male stay times results in all male dung flies having the same reproductive opportunity. In other words, the exponential distribution is optimal for each male fly.

Studies of industrial melanism in moths provide a nice example of testing the assumption that there is heritable variation for a trait, as described by Reznick and Travis (1996). Trait values in different populations and in the same population at different points in time can be compared to establish the existence of available variation. Once this has been established, one way to assess the heritability of this variation is to determine whether there is a genetic basis for the trait. This can be accomplished by, e.g., transferring to a common environment samples from populations that differ in mean values for the trait. If differences persist, this suggests that the trait has genetic influences (Reznick and Travis, 1996).

Lastly, to establish that an optimality model represents all important evolutionary influences, all of the constraints that are accounted for in the model must be relatively universal constraints. Recall that optimality models always predict local optima: the best possible trait values within some range of alternatives. According to biological parlance, if the range of alternatives is limited only by universal constraints - broad constraints arising from physical and biological possibility (Maynard Smith et al., 1985), then these constraints are not

outcome; independent tests of assumptions; and variety of evidence. Testing the components of an optimality model is an instance of the second type of confirmation. These components should be carefully tested for the weak use of an optimality model as well, for this assures that the optimality model accurately represents the selection dynamics. Yet the strong use of an optimality model creates a further reason to test the model's components, for undetected inaccuracies can conceal the failure of optimality, which undermines the success of the strong use. 
taken to constitute non-selective evolutionary factors. Otherwise, selection could not possibly ever be the only important evolutionary factor, since all optima are subject to universal constraints. In contrast, if the range of alternatives is significantly constrained by features peculiar to the specific population under investigation, or possibly to the species or higher taxon, then the sources of these constraints are considered to be important evolutionary factors in their own right. ${ }^{5}$ These include, e.g., particular developmental, phylogenetic, and genetic influences on evolution. Because optimality models do not represent such nonselective influences (see discussion in Section 1), the strong use of an optimality model is only successful if all significant constraints are universal in nature.

Rigorously testing each component of an optimality model, including the optimizing assumption, and considering the sources of constraints built into the model are necessary to protect the strong use of optimality models from the criticisms of the optimality approach that have arisen from concerns regarding adaptationism. First, carefully testing the components of an optimality model and the optimizing assumption blocks Gould and Lewontin's (1979) charge that the optimality approach is merely an exercise in ingenuity, where optimality is assumed and imaginative stories are cooked up to support this assumption. Second, accounting only for universal constraints in the model ensures that the model represents all important evolutionary factors, namely natural selection. For this to be true, constraints built into the model must not covertly introduce place-holders for significant non-selective influences, such as developmental or genetic constraints.

The breadth of success enjoyed by the strong use of optimality models is thus limited by the degree to which adaptationism is true, where adaptationism is taken to be the claim that selection is the only important influence on most evolutionary outcomes. This conception of adaptationism is like Orzack and Sober's (1994) definition of strong adaptationism and Godfrey-Smith's (2001) definition of empirical adaptationism. ${ }^{6}$ This is as it should be. The

\footnotetext{
${ }^{5}$ See, for example, Maynard Smith et al.'s (1985) discussion of developmental constraints.

${ }^{6}$ Other distinct conceptions of adaptationism have been formulated; see Godfrey-Smith (2001) and
} 
strong use of an optimality model involves the claim that the model's representation of selection dynamics provides an accurate picture of the important influences on the evolutionary outcome in question. The breadth of success enjoyed by the strong use thus depends upon how often selection is the only important evolutionary influence. This articulation of the strong use of optimality models preserves the tight relationship between optimality models and adaptationism that is assumed in most philosophical discussions of the optimality approach, and that is directly proposed by Orzack and Sober (1994).

However, this tight relationship does not hold for weaker uses of optimality models. As discussed in Section 1, an optimality model may succeed in what I term its weak use, regardless of whether natural selection is the only important evolutionary factor. The debate over adaptationism is relevant to this weak use of optimality models only via the methodological points that optimality models should reflect significant constraints and that the influence and dynamics of selection must be carefully evaluated. An implication of this separation between the weak use of optimality models and the debate over adaptationism is that the successful employment of an optimality model in its weak use does not provide evidence in favor of adaptationism. If the weak use of optimality models enjoys widespread success, then this demonstrates only that selection is often one evolutionary factor, potentially among many. This is not enough to generate support for adaptationism, as it is understood here.

Orzack and Sober (1994) offer the most systematic discussion of evaluating the importance of selection. They distinguish three distinct claims that might be made about the role of selection:

(U) Natural selection played some role in the evolution of $T$.

(I) Natural selection was an important cause of the evolution of $T$.

(O) Natural selection is a sufficient explanation of the evolution of $T$, and $T$ is locally Lewens's paper in this volume. I do not discuss these alternate conceptions, except the brief comment in footnote 7 . 
optimal $(1994,362)$.

Adaptationism is claim that $(\mathrm{O})$ is true for most nonmolecular traits. If the strong use of optimality models enjoys wide success, according to the standards of success articulated by Orzack and Sober and discussed here, then this would vindicate adaptationism. In contrast, the success of an optimality model in its weak use ensures only that selection dynamics played some role in the evolution of the trait. The broad success of the weak use of optimality models thus underwrites only the claim that $(\mathbf{U})$ is true for many traits, which falls short of adaptationism.

Distinguishing between the weak and strong uses of optimality models thus clarifies the relationship between the optimality approach and adaptationism. Most biologists accede that concerns over adaptationism warrant certain methodological correctives (e.g., Maynard Smith, 1982, 5). This basic point applies to the weaker uses of optimality models, as well as to the strong use. The further ideas that the justification for optimality modeling is adaptationism and that optimality models can be used to generate support for adaptationism (Orzack and Sober, 1994) apply only to the strong use of optimality models, wherein an optimality model is purported to represent the only important evolutionary factor, viz. natural selection. The legitimacy and usefulness of weaker uses of optimality models thus are not threatened by the fate of adaptationism, whatever that fate may be. The optimality approach can be used to represent the dynamics of selection without placing any bets on the relative importance of selection compared to other, non-selective evolutionary factors such as genetic drift and developmental, phylogenetic and genetic constraints.

\section{Weak, Not Powerless}

At the start of this paper, I claimed that optimality modeling could be decoupled from adaptationism. Up to this point, I have argued that only the strong use of optimality models 
requires a commitment to adaptationism for its justification, and that population biologists often intend weaker uses for the optimality models they construct. Nonetheless, one might be concerned that the optimality approach is of little importance to population biology when it is prised apart from the issue of adaptationism. Biologists may often intend or seem to intend weaker uses of optimality models, but perhaps they are mistaken in doing so, or perhaps I am mistaken in representing their intentions in this way. In the weak use I have focused on, optimality models are used to represent selection dynamics but not to establish that selection is the only important evolutionary factor. But because optimality models do not represent non-selective evolutionary factors, it seems that the role of an optimality model in its weak use is simply to represent a single evolutionary factor - selection - which may be of quite limited significance. Of what interest are optimality models decoupled from adaptationism, if they have such a narrowly defined role?

It would be mistaken to think that the optimality approach is by itself a sufficient approach to modeling evolutionary change. The possibility of non-selective evolutionary factors - developmental, phylogenetic and genetic constraints; drift; etc.-must be considered, even if the consideration of these factors leads them to be eliminated as possibilities. Because the potential of non-selective factors warrants investigation, the optimality approach is not by itself a sufficient approach to modeling evolutionary change. ${ }^{7}$ This is so at least when non-selective factors are suspected to have shaped the outcome. The optimality approach is simply one useful approach among others-but it is useful nonetheless. When selection influences an evolutionary outcome, the nature of this influence bears investigation. Criticisms of adaptationism notwithstanding, selection is a significant factor in the evolution of many traits that receive attention from biologists. The optimality approach provides a

\footnotetext{
${ }^{7}$ An anonymous referee has pointed out that an appreciation for the weaker uses of optimality models might be used to argue for methodological adaptationism - that the best approach to studying biological systems is to look for adaptations (Godfrey-Smith, 2001). I agree that this is possible, but it is not a road I would take. As this discussion indicates, I think a myopic focus on adaptations is misguided; non-selective influences on evolution warrant investigation in their own right.
} 
tractable way to model the dynamics of selection. Optimality models represent the nature of selection's influence, and they are tractable in virtue of their neglect of non-selective factors like drift and the details of genetic transmission.

Indeed, optimality models can be useful even when non-selective evolutionary factors cause observed trait values to vary significantly from predicted values. The failure or quite limited success of a carefully constructed optimality model itself has information to offer about the evolutionary scenario at hand, for this suggests that additional, non-selective factors are unaccounted for (Seger and Stubblefield, 1996; Godfrey-Smith, 2001). If a wellconfirmed fitness function indicates that natural selection would result in the population having a particular trait value, and yet the measured trait value differs markedly from this predicted value, one has reason to wonder what is responsible for the discrepancy. A pluralist perspective regarding evolutionary change allows for many possible causes of deviation from the predicted optimum. The environment might have recently undergone a change relevant to the trait's fitness; some genetic, developmental or phylogenetic constraint may exist; the trait may be subject to an unexpected fitness tradeoff; drift may have significantly affected the outcome; etc. The failure of an optimality model's prediction leads one to investigate such possibilities and may suggest particularly likely influences. This facilitates insight into the influence of non-selective factors on the target evolutionary outcome.

There is another, related way in which the failure of an optimality model can provide insight into evolution. The comparison between the actual result of evolution and the theoretical result of natural selection acting alone can itself be of interest, even apart from leading to an improved recognition of non-selective evolutionary factors. Orzack and Sober (1994) suggest something along these lines. They think that optimality models are important even when they fail, since "[a] fundamental contribution of such models is that they describe what organisms should do in particular instances" $(1994,378)$. Optimality models detail what the result would be if natural selection had been responsible for the evolution of the trait in 
question. A comparison between this and the actual result of evolution yields insight into the role of selection in that particular instance of evolution.

These ideas are illustrated by Orzack's (1986) analysis of an optimality model for the sex ratio of Nasonia vitripennis, a parasitic wasp. The optimality model, developed by Werren (1980), predicts that the optimal sex ratio varies according to population structure. When the population is subdivided so that siblings compete for mates, the sex ratio will be femalebiased, whereas wide dispersal before breeding creates the opportunity for outcrossing, and sex ratios will be much more equitable (Hamilton, 1967; Werren, 1980). For the wasp $N$. vitripennis, these two scenarios are respectively realized $(a)$ when a female visits a host that has not been previously parasitized, and $(b)$ when eggs are laid in a host already containing a wasp brood. The optimality model thus predicts that the proportion of males in the second brood in a host should directly correlate with the existence and size of the first brood. A large first brood provides the opportunity for the second brood to outbreed, whereas a small or nonexistent first brood results in a second brood that will largely inbreed.

However, Orzack (1986) finds that these predictions fit the data poorly. Sex ratios in previously parasitized hosts differ significantly from the predicted ratio, and the observed sex ratios also vary significantly. Figure 1 plots some of Orzack's data against the curve predicted by the optimality model. Orzack proposes a "nonoptimal model" that would account for this variability. According to this model, a female visiting a host can potentially detect a pheromonal signal left by an earlier egg-laying female. When a female detects this signal, the sex ratio of her eggs is altered to increase proportion of males, but this is not accomplished with great precision. Some variation in sex ratio is thus introduced. Further, if the first brood in the host is small, then the pheromonal signal will be reduced. The second female visiting is less able to detect the signal, and the sex ratio of her eggs remains female-biased. Orzack suspects that this accounts for how sex-ratio control in $N$. vitripennis fails to conform to the pattern expected on the basis of the optimality approach. Of course, evidence must 


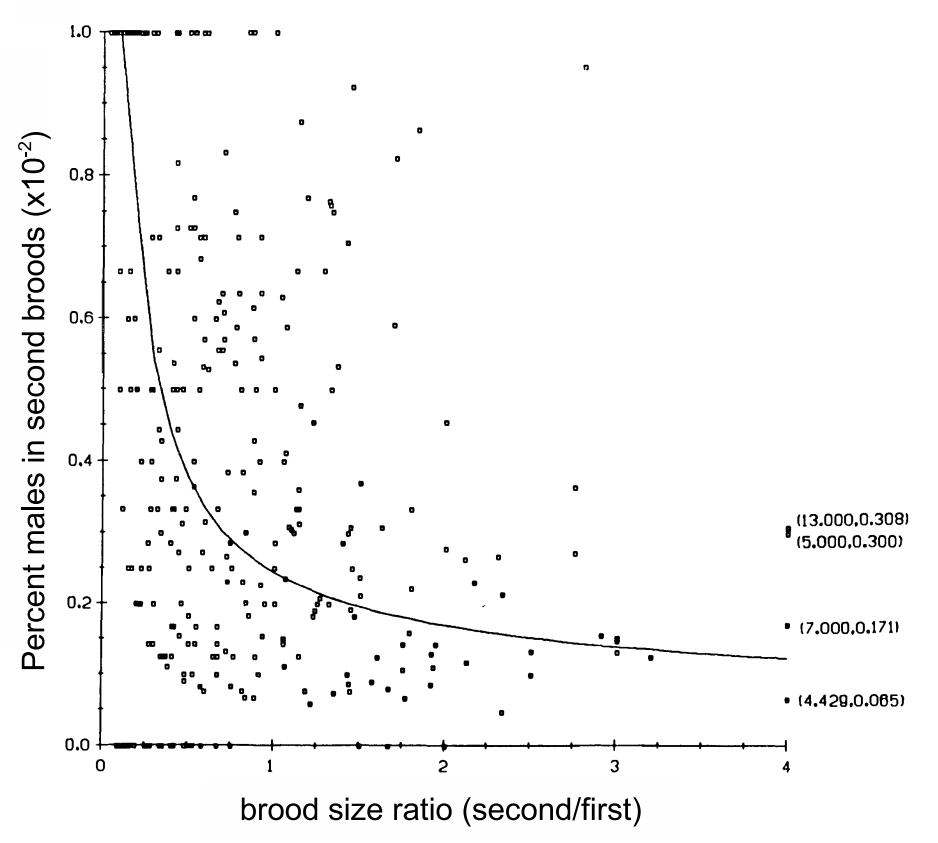

Figure 1: Orzack's (1986) comparison between the sex-ratio curve predicted by Werren's (1980) optimality model and the sex ratios found for different ratios of second-brood size to first-brood size. This figure compiles the data from several experiments on two strains of Nasonia vitripennis, the Ithaca wildtype and scarlet eye strains.

be gathered in order for this nonoptimal model to be confirmed.

Despite the failure of the optimality model for the wasps' sex ratio, Orzack emphasizes the importance of developing the model:

I believe that the local mate competition model of Hamilton (1967) gives insight into the potential direction of sex-ratio evolution and into possible selection pressures on sex-ratio modification in response to inbreeding and to variation in population structure $(1986,355)$.

Orzack proceeds to cite the patterns in his data that do not conform to Hamilton's insights and points out that these patterns may conflict with the optimality model of sex-ratio evolution, and that they must be accounted for by a successful theory of sex ratio in the wasp 
under study.

This work exemplifies the ways in which a well-motivated optimality model can be valuable, even when the model's predictions are not born out. The optimality model of N. vitripennis's sex ratio offers insight into the selection pressures involved, and it directs attention toward the patterns that do not conform to expectations. These contributions - insight into the selection dynamics and focusing attention toward non-selective factors - are made by optimality models in their weak use of representing the role of selection. This is true when the predicted and observed trait values differ widely from each other, as well as when there is closer correspondence to the model's predictions.

The strong use of optimality models, where a model is taken to represent all important evolutionary factors, is thus not the only important role that optimality models play. In this paper I focus on one prominent weaker use of optimality models, where an optimality model is simply used to represent the role of selection in bringing about an evolutionary outcome, without the claim that this is the only important influence on the outcome. This weak use is common in population biology, and for good reason: it offers a tractable way to gain insight into the dynamics of selection and the evolutionary role played by these dynamics. Other weaker uses of optimality models are also valuable, including using optimality models to initially explore the plausibility of adaptive hypotheses. This use is especially valuable when little is known about the population under investigation. Because such weaker uses of optimality models do not involve claims about the relative importance of selection, the optimality approach is able remain a well-founded and important tool of population biology, regardless of the fate of adaptationism. 


\section{Acknowledgments}

This paper has benefitted from the insights of Elliott Sober, Joan Roughgarden and the members of her lab, Peter Godfrey-Smith, Patrick Forber, and two anonymous referees for Biology and Philosophy.

\section{References}

Brandon, R. N. and Rausher, M. D. (1996). Testing adaptationism: A comment on Orzack and Sober. The American Naturalist, 148(1):189-201.

Godfrey-Smith, P. (2001). Three kinds of adaptationism. In Orzack, S. H. and Sober, E., editors, Adaptationism and Optimality, Cambridge Studies in Philosophy and Biology, chapter 11, pages 335-357. Cambridge University Press, Cambridge, UK.

Gould, S. J. and Lewontin, R. (1979). The spandrels of San Marco and the panglossian paradigm: A critique of the adaptationist programme. Proceedings of the Religious Society of London, 205:581-598.

Hamilton, W. (1967). Extraordinary sex ratios. Science, 156:477-488.

Hofbauer, J. and Sigmund, K. (1998). Evolutionary Games and Replicator Dynamics. Cambridge University Press, Cambridge, UK.

Lewens, T. (2009). Seven types of adaptationism. Biology and Philosophy.

Lloyd, E. A. (1988). The structure and confirmation of evolutionary theory. Princeton University Press, Princeton, NJ.

Maynard Smith, J. (1982). Evolution and the Theory of Games. Cambridge University Press, Cambridge, UK.

Maynard Smith, J., Burian, R., Kauffman, S., Alberch, P., Campbell, J., Goodwin, B., Lande, R., Raup, D., and Wolpert, L. (1985). Developmental constraints and evolution. Quarterly Review of Biology, 60(3):265-287.

Orzack, S. H. (1986). Sex-ratio control in a parasitic wasp, Nasonia vitripennis. II. Experimental analysis of an optimal sex-ratio model. Evolution, 40(2):341-356.

Orzack, S. H. and Sober, E. (1994). Optimality models and the test of adaptationism. The American Naturalist, 143(3):361-380.

Orzack, S. H. and Sober, E. (1996). How to formulate and test adaptationism. The American Naturalist, 148(1):202-210.

Parker, G. (1970). The reproductive behavior and the nature of sexual selection in 
Scatophaga stercoraria L. (Diptera: Scatophagidae): Ii. The fertilization rate and the spatial and temporal relationships of each sex around the site of mating and oviposition. The Journal of Animal Ecology, 39(1):205-228.

Reznick, D. and Travis, J. (1996). The empirical study of adaptation in natural populations, chapter 8, pages 243-289. Academic Press, San Diego, CA.

Seger, J. and Stubblefield, J. W. (1996). Optimization and adaptation. In Rose, M. R. and Lauder, G. V., editors, Adaptation, chapter 3, pages 93-123. Academic Press, San Diego, CA.

Werren, J. (1980). Sex ratio adaptations to local mate competition in a parasitic wasp. Science, 208:1157-1159. 